A flexible method to calculate the distributions of discretization errors in operator-split codes with stochastic noise in problem data

X. Chen, J. M. Connors, C. H. Tong

January 31, 2014 
This document was prepared as an account of work sponsored by an agency of the United States government. Neither the United States government nor Lawrence Livermore National Security, LLC, nor any of their employees makes any warranty, expressed or implied, or assumes any legal liability or responsibility for the accuracy, completeness, or usefulness of any information, apparatus, product, or process disclosed, or represents that its use would not infringe privately owned rights. Reference herein to any specific commercial product, process, or service by trade name, trademark, manufacturer, or otherwise does not necessarily constitute or imply its endorsement, recommendation, or favoring by the United States government or Lawrence Livermore National Security, LLC. The views and opinions of authors expressed herein do not necessarily state or reflect those of the United States government or Lawrence Livermore National Security, LLC, and shall not be used for advertising or product endorsement purposes.

This work performed under the auspices of the U.S. Department of Energy by Lawrence Livermore National Laboratory under Contract DE-AC52-07NA27344. 


\title{
A flexible method to calculate the distributions of discretization errors in operator-split codes with stochastic noise in problem data
}

\author{
Xiao Chen, Jeffrey M. Connors and Charles Tong *
}

January 29, 2014

\begin{abstract}
This report investigates a technique to calculate the distributions of discretization errors for a model of advection-diffusion-reaction with stochastic noise in problem data. The focus is on operator-split discretization methods. The error is decomposed into components due to the splitting and due to the discretization within each component. We present a method to estimate the distributions of the total discretization error for a quantity of interest as well as the aforementioned error components. These computations require information about the propagation of uncertainty within and between code modules, for which purpose we use a flexible code framework for uncertainty quantification. Computational examples illustrate that in certain cases the distribution of numerical error can have a significant impact on the code output. Using information about the components of the numerical error, decisions can be made regarding discretization parameters to efficiently reduce the impact of numerical error on the distribution of model outputs.
\end{abstract}

\section{Introduction}

Uncertainty quantification (UQ) for multi-physics simulation seeks to provide insight into the meaning of computational model outputs as they relate to the prediction of physical phenomena. Stochastic noise in model data induces a statistical distribution in model outputs, which may often be quantified approximately using numerical techniques. Many factors must be accounted for to explain the ensuing discrepancies with respect to observations. One factor is the use of discretization techniques for the equations representing the physical processes. These techniques introduce numerical errors that may significantly

* Lawrence Livermore National Laboratory, Center for Applied Scientific Computing, Livermore, CA, 94551, USA. This work was performed under the auspices of the U.S. Department of Energy by Lawrence Livermore National Laboratory under Contract DE-AC52-07NA27344. LLNL-TR-xxxxxx. 
influence the distribution of model outputs and potentially provide a poor representation of the underlying multi-physics model equations.

Multi-physics codes are often constructed by coupling together code modules that are highly-optimized for the resolution of physical sub-processes. In the current context, for example, we consider coupling three modules that approximate the advection, diffusion and reaction processes, respectively, via an operator-splitting technique to create a multi-physics model for the full advection-diffusion-reaction system. In addition to the distribution of the total discretization error for a multi-physics model, it is desirable to understand how the discretization errors for each separate module and the splitting error contribute to the total error budget. Then decisions can be made regarding the allocation of computational resources to control the global error budget. This report focuses on the quantification of the distribution of discretization errors for a model of advection-diffusion-reaction. These computations require information about the propagation of uncertainty within and between code modules, for which purpose we use a special, flexible code framework.

In order to model some physical process that is believed to essentially be a coupled advection-diffusion-reaction process, one might hypothesize that the following model, for example, closely resembles reality in some open spatial domain $S \subset \mathbb{R}^{d}$ :

$$
\begin{aligned}
\partial_{t} c+\nabla \cdot(\mathbf{v}(x) c)+R c & =D \Delta c,(x, t) \in S \times(0, T], \\
c & =g,(x, t) \in \partial S \times(0, T], \\
c(x, t=0) & =c_{0}(x), x \in S
\end{aligned}
$$

where $\mathbf{v}(x)$ is a prescribed flow field, $R$ is a reaction coefficient and $D>0$ is a diffusion coefficient. The solution is $c: \bar{S} \times[0, T] \rightarrow \mathbb{R}$, which may represent the concentration of some chemical species. Consider that the flow field, the reaction coefficient and the diffusion coefficient may be uncertain and are modeled as independent, second-order random variables (r.v-s) over a probability space $(\Omega, \Theta, P)$, where $\Omega$ is the sample space, $\Theta$ is a $\sigma$-algebra on $\Omega$ and $P$ is a probability measure. Then $c$ is a dependent r.v. and we require that the above SPDE problem is solvable almost surely.

Various methods exist to model the propagation of uncertainty and to discretize the PDE. Ultimately, some finite-dimensional representation $\tilde{c} \approx c$ is calculated, which could be considered as the model for the physical process. In this case, since $\tilde{c}$ will not exactly represent reality, there is some model form error $\epsilon(\tilde{c})$ such that the real distribution as observed by experiment (in the absence of observational errors) is $\tilde{c}+\epsilon(\tilde{c})$.

Recall that for a r.v. $X$ defined on the probability space $(\Omega, \Theta, P)$, the expected (mean) value $E[X]$ is

$$
E[X]=\int_{\Omega} X(\omega) d P(\omega) .
$$

Our goal is to obtain more information about the structure of $\epsilon(\tilde{c})$ by hypothe- 
sizing that

$$
E\left[\|\epsilon(c)\|_{L^{\infty}(\bar{S} \times[0, T])}\right]<E\left[\|\epsilon(\tilde{c})\|_{L^{\infty}(\bar{S} \times[0, T])}\right] .
$$

Our meaning is that we expect $c$ may be a better model of reality $(c+\epsilon(c))$ than $\tilde{c}$ since $\tilde{c}$ is derived by approximating $c$. Under this assumption, it is useful to consider

$$
c=(c-\tilde{c})+\tilde{c}=e+\tilde{c} .
$$

Assume that we are interested in a measurement $\mathcal{M}(c)$ of $c$ and that the measurement may be written in the form

$$
\mathcal{M}(c)=\int_{\Omega} G(x) c(x, t=T) d x,
$$

where $G(x)$ is known as the generalized Green's function for the measurement. Then we seek to estimate the discrepancy

$$
e_{\mathcal{M}}=\mathcal{M}(c)-\mathcal{M}(\tilde{c}),
$$

which is a r.v. by definition.

Specifically, we are interested in the case when the reaction, diffusion and advection processes are separated by operator-splitting. We assume that a code module for each process is available, and that they are sequentially coupled in some manner to evolve $\tilde{c}$ in time. The propagation of stochastic noise may be handled using a hybrid method of uncertainty quantification, whereby each discrete degree of freedom is represented as a random variable with a truncated polynomial chaos (PC) expansion that accounts for the global r.v. -s. The stochastic analysis is discussed in Section 2. The internal propagation of uncertainty may either be intrusive or not.

The discrepancy $e$ will generally be affected by (1) the splitting approximation; (2) the application of numerical methods for the advection, diffusion and reaction subproblems; and (3) truncation of the PC expansion used to represent the stochastic process. In order to obtain more information about the effects of the choices of splitting method and numerical methods, we further decompose

$$
e=\left(c-c_{s}\right)+\left(c_{s}-\tilde{c}\right) .
$$

Here, $c_{s}$ is obtained by splitting the operators in the original problem (1.1) for $c$ before choosing space and time discretizations for the resulting subproblems or truncating the PC expansion.

We consider a first-order sequential splitting method to define $c_{s}$. Let $\Delta t=$ $T / N_{T}$ be a time step size for some $N_{T} \in \mathbb{N}$, and $t^{n}=n \Delta t$ for $n=0,1, \ldots, N_{T}$. We first define the split solution at the discrete times. Let $c_{s}\left(x, t^{n}\right)=c_{s}^{n}(x)$, where $c_{s}^{0}=c_{0}$ and the functions $c_{s}^{n+1}$ for $n \geq 0$ are found from the following procedure.

- Step 1: Solve for $c_{a}$ satisfying

$$
\begin{aligned}
\partial_{t} c_{a}+\nabla \cdot\left(\mathbf{v}(x) c_{a}\right) & =0,(x, t) \in S \times\left(t^{n}, t^{n+1}\right], \\
c_{a} & =g_{a},(x, t) \in \partial S \times\left(t^{n}, t^{n+1}\right], \\
c_{a}\left(x, t=t^{n}\right) & =c_{s}^{n}(x), x \in S .
\end{aligned}
$$


- Step 2: Solve for $c_{d}$ satisfying

$$
\begin{aligned}
\partial_{t} c_{d} & =D \Delta c_{d},(x, t) \in S \times\left(t^{n}, t^{n+1}\right], \\
c_{d} & =g_{d},(x, t) \in \partial S \times\left(t^{n}, t^{n+1}\right], \\
c_{d}\left(x, t=t^{n}\right) & =c_{a}\left(x, t^{n+1}\right), x \in S .
\end{aligned}
$$

- Step 3: Solve for $c_{r}$ satisfying

$$
\begin{aligned}
\partial_{t} c_{r}+R c_{r} & =0,(x, t) \in \bar{S} \times\left(t^{n}, t^{n+1}\right], \\
c_{r}\left(x, t=t^{n}\right) & =c_{d}\left(x, t^{n+1}\right), x \in S .
\end{aligned}
$$

- Step 4: $\operatorname{set} c_{s}^{n+1}(x)=c_{r}\left(x, t^{n+1}\right)$.

- Step 5: set $n=n+1$ and repeat Step 1 - Step 4 until $n=N_{T}$.

Then $c_{s}(x, t)$ may be defined as some suitable extension of $\left\{c_{s}^{n}(x)\right\}_{n=0}^{n=N_{T}}$ in time, such as by using interpolation. Note that we allow for the reaction and advection-diffusion substeps to use different boundary conditions. These should be consistent with what is implemented in the code to generate $\tilde{c}$.

The corresponding decomposition for $e_{\mathcal{M}}$ is assigned the following meaning:

$$
\begin{aligned}
\text { splitting error: } e_{\text {split }} & =\mathcal{M}(c)-\mathcal{M}\left(c_{s}\right) ; \\
\text { module discretization error: } e_{\text {disc }} & =\mathcal{M}\left(c_{s}\right)-\mathcal{M}(\tilde{c}) .
\end{aligned}
$$

The module discretization error may in turn be further decomposed:

$$
e_{d i s c}=e_{A}+e_{D}+e_{R}+e_{\text {interp }}+e_{I C} .
$$

Here $e_{A}, e_{D}$ and $e_{R}$ are the error contributions from the advection, diffusion and reaction module discretizations, respectively. If the grids differ between the modules then $e_{\text {interp }}$ may be included as the error contribution from interpolating between grids. The error from approximating initial data is $e_{I C}$.

The distributions of the error components will be estimated using adjoint methods, described below. The effect of the splitting is then determined by the relationship $e_{\mathcal{M}}=e_{\text {split }}+e_{\text {disc }}$ (just add (1.14) and (1.15) to get (1.5)). We will begin by presenting the flexible-UQ method to track the propagation of uncertainty in the error within and between each module, then explain the adjoint methods to calculate estimates of the error components.

\section{Flexible-UQ Formulation}

Given a second-order random variable $X$ defined on the probability space $(\Omega, \Theta, P)$, the variance of $X$ is denoted by $\operatorname{Var}(X)$, satisfying

$$
\operatorname{Var}(x)=E\left[X^{2}\right]-(E[X])^{2},
$$


and the standard deviation $\sigma \geq 0$ is $\sigma=\sqrt{\operatorname{Var}(X)}$. The random variable $X$ may be expressed using an expansion in terms of polynomials over the stochastic space that are orthogonal with respect to the density of $X$ :

$$
X=X_{0}+\sum_{j_{1}=1}^{\infty} X_{j_{1}} \Psi_{1}\left(\xi_{j_{1}}\right)+\sum_{j_{1}=1}^{\infty} \sum_{j_{2}=1}^{j_{1}} X_{j_{1}, j_{2}} \Psi_{2}\left(\xi_{j_{1}}, \xi_{j_{2}}\right)+\ldots
$$

There are $m$ dimensions for the random variable, $\xi=\left(\xi_{1}, \ldots, \xi_{m}\right)$. This PC expansion is then truncated to some order $p$. For convenience, we use a singleindex format for the truncated PC expansion with $P+1=(p+m) ! /(p ! m !)$ terms:

$$
\hat{X}=\sum_{j=0}^{P} X_{j} \Psi_{j}(\xi)
$$

If $X, Y$ are r.v-s, we denote by $\langle X Y\rangle$ the integral of $X Y$ over the entire stochastic space, with appropriate extension to products of more r.v-s . The expected value and variance for $\hat{X}$ are

$$
\begin{aligned}
E[\hat{X}] & =X_{0} \\
\operatorname{Var}(\hat{X}) & =\sum_{j=0}^{P} X_{j}^{2}\left\langle\Psi_{j}^{2}\right\rangle .
\end{aligned}
$$

We will assume for now that all the r.v-s being discussed have the same density. The independent r.v-s are $\mathbf{v}=\left\langle v_{1}, \ldots, v_{d}\right\rangle, D$, and $R$. Uncertainties are propagated globally by using a PC expansion with $d+2$ r.v-s to order $p$ so that there are $P_{g}=(d+2+p) ! /((d+2) ! p !)$ coefficients. The calculation of numerical errors will use information about the propagation of solution information through each module. Since the individual modules do not make use of all of the global r.v-s some way to propagate the global uncertainty through the local module is needed. For this purpose we make use of the technique described in [1]. The degrees of freedom for the global PC expansion may be calculated from the output of a module by running successively with different input configurations.

Internally, we require $P_{a}=(d+p) ! /(d ! p !)$ expansion coefficients for the advection module and $P_{d r}=(1+p) ! /(p !)=p+1$ expansion coefficients for the diffusion and reaction modules. The details of how to specify the input configurations are provided in [1]; we instead focus here on the computation of numerical errors. We shall see that these calculations require the ability to determine these input configurations and properly distinguish between local and global propagation of uncertainty.

\section{Adjoint Method to Calculate $e_{\mathcal{M}}$ and $e_{\text {disc }}$}

In this section we describe the method to calculate $e_{\mathcal{M}}$ and $e_{d i s c}$ as r.v-s using adjoint methods. First, we introduce the adjoint problem for $e$. The Green's 
function data $G$ is used as the final time data for the adjoint problem:

$$
\begin{aligned}
-\partial_{t} \phi-\mathbf{v} \cdot \nabla \phi+R \phi & =D \Delta \phi,(x, t) \in S \times[0, T), \\
\phi & =0,(x, t) \in \partial S \times[0, T), \\
\phi(x, t=T) & =G(x), x \in S .
\end{aligned}
$$

We will consider finite volume spatial discretizations, though our method applies more generally. Assume a uniform, Cartesian spatial grid $S_{h}$ where $h>0$ is the grid size. Given a grid element $\mathcal{K}$ with center $x=\left(x_{1}, \ldots, x_{d}\right)$, the grid element is defined as $\mathcal{K}=\prod_{i=1}^{d}\left[x_{i}-h / 2, x_{i}+h / 2\right]$. Let $t^{n}$ for $n=0,1, \ldots, N$ be discrete time levels for the purposes of time discretization. Given an arbitrary function $v$, assume that some space and time discretization is applied to approximate $v$. We denote by $v_{h}$ a continuum representation for the ensuing approximation, which takes the form of a polynomial on each grid element in space and in time is a polynomial on each discrete time interval. We assume global continuity of $v_{h}$ in time, and in fact $\tilde{c} \in C^{0}\left(0, T ; L^{2}(\bar{S})\right)$, but we allow $v_{h}$ to be discontinuous across grid element interfaces. We denote the interior trace of a function $v$ restricted to an element by $v_{-}$, and the exterior trace for nonboundary edges by $v_{+}$. The jump in values across an interface is $[v]=v_{+}-v_{-}$ and the average is $\{v\}=\left(v_{+}+v_{-}\right) / 2$. Furthermore, we define for convenience

$$
\partial \mathcal{K}^{+}=\left\{x \in \partial \mathcal{K}: \mathbf{v}(x) \cdot \hat{n}_{\mathcal{K}}>0\right\} .
$$

The truncated PC expansion for an arbitrary function $v$ is $\hat{v}$. Thus, $\hat{v}_{h}$ is fully-discrete, whereas $v_{h}$ is only discrete in space and time. The approximation $\tilde{c}$ of the advection-diffusion-reaction problem is taken to be $\tilde{c}=\hat{c_{h}}$.

The equation for $e_{\mathcal{M}}$ is given by (see e.g. [3, 2] for details):

$$
\begin{aligned}
& e_{\mathcal{M}}=\int_{0}^{T} \int_{S}\left(-\partial_{t} \tilde{c}-\nabla \cdot(\mathbf{v} \tilde{c})+D \Delta \tilde{c}-R \tilde{c}\right) \phi d x d t \\
& +\sum_{\mathcal{K} \in \Omega_{h}} \int_{0}^{T} \int_{\partial \mathcal{K}+\backslash \partial \Omega}\left(D \phi\left(\hat{n}_{\mathcal{K}} \cdot \nabla[\tilde{c}]\right)-D\left(\hat{n}_{\mathcal{K}} \cdot \nabla \phi\right)[\tilde{c}]-\left(\mathbf{v} \cdot \hat{n}_{\mathcal{K}}\right) \phi[\tilde{c}]\right) d(\partial S) d t \\
& \quad-\int_{0}^{T} \int_{\partial S} D(\hat{n} \cdot \nabla \phi)(g-\tilde{c}) d(\partial S) d t+\int_{S}\left(c_{0}-\tilde{c}(t=0)\right) \phi(t=0) d x .
\end{aligned}
$$

There will be a limit to our knowledge of the numerical error due to inexact knowledge of the adjoint solution, including uncertainty and discretization errors. Denote by $\tilde{\phi}$ some fully-discrete approximation to $\phi$ and let $\phi=\tilde{\phi}+\phi_{H O}$.

Note that the right hand side of (3.4) may be written in the form

$$
e_{\mathcal{M}}=\mathcal{F}(\tilde{c}, \phi)+\mathcal{L}(\phi)
$$

where $\mathcal{F}$ is bilinear and $\mathcal{L}$ is linear. It follows that

$$
e_{\mathcal{M}}=(\mathcal{F}(\tilde{c}, \tilde{\phi})+\mathcal{L}(\tilde{\phi}))+\left(\mathcal{F}\left(\tilde{c}, \phi_{H O}\right)+\mathcal{L}\left(\phi_{H O}\right)\right)
$$


The first two terms on the right hand side are fully computable. The other terms will be neglected for the purposes of this report, i.e. they are assumed to be negligible. This assumption will hold if the relative accuracy of the approximation $\tilde{\phi}$ is very good, i.e. if $\left|\phi_{H O}\right| \ll|\phi|$, as explained in the deterministic case in e.g. $[3,2]$.

So far, the flexible-UQ framework is employed to generate the $\mathrm{PC}$ expansion for $\tilde{c}$, which is used at each time step and every spatial degree of freedom to calculate the error approximation. We shall see that the remaining components of the discretization error will also require the use of the flexible-UQ framework.

Before describing the equation for $e_{d i s c}$, we first introduce some notation. We let $\tilde{c}_{a}$ be some discrete approximation to the solution of (1.6)-(1.8) (usually just linear in time on the local time interval). Similarly, let $\tilde{c}_{d}$ and $\tilde{c}_{r}$ represent the discrete solutions of the diffusion and reaction modules.

On each local time interval we will derive local discretization error estimates by using the adjoint to (1.6)-(1.13). This adjoint problem may be expressed as follows, with $\phi_{s}^{N_{T}}=G$.

- Step 1: Solve for $\phi_{r}$ satisfying

$$
\begin{aligned}
-\partial_{t} \phi_{r}+R \phi_{r} & =0,(x, t) \in \bar{S} \times\left[t^{n}, t^{n+1}\right), \\
\phi_{r}\left(x, t=t^{n+1}\right) & =\phi_{s}\left(x, t^{n+1}\right), x \in S .
\end{aligned}
$$

- Step 2: Solve for $\phi_{d}$ satisfying

$$
\begin{aligned}
-\partial_{t} \phi_{d} & =D \Delta \phi_{d},(x, t) \in S \times\left[t^{n}, t^{n+1}\right), \\
\phi_{d} & =0,(x, t) \in \partial S \times\left(t^{n}, t^{n+1}\right], \\
\phi_{d}\left(x, t=t^{n+1}\right) & =\phi_{r}\left(x, t^{n}\right), x \in S .
\end{aligned}
$$

- Step 3: Solve for $\phi_{a}$ satisfying

$$
\begin{aligned}
-\partial_{t} \phi_{a}-\mathbf{v} \cdot \nabla \phi_{a} & =0,(x, t) \in S \times\left[t^{n}, t^{n+1}\right), \\
\phi_{a} & =0,(x, t) \in \partial S \times\left(t^{n}, t^{n+1}\right], \\
\phi_{a}\left(x, t=t^{n+1}\right) & =\phi_{d}\left(x, t^{n}\right), x \in S .
\end{aligned}
$$

- Step 4: $\operatorname{set} \phi_{s}^{n}(x)=\phi_{a}\left(x, t^{n}\right)$.

- Step 5: set $n=n-1$ and repeat Step 1 - Step 3 until $n=0$.

The expression for $e_{\text {disc }}$ is derived by starting on the last time step, noting that $\left(c_{s}-\tilde{c}\right)(t=T)=\left(c_{r}-\tilde{c}_{r}\right)(t=T)$ and recalling that $\phi_{s}(t=T)=\phi_{r}(t=$ 
$T)=G$. Thus, we have

$$
\begin{gathered}
e_{d i s c}=\int_{S} G\left(c_{s}-\tilde{c}\right)(t=T) d x=\int_{S} \phi_{r}(t=T)\left(c_{r}-\tilde{c}_{r}\right)(t=T) d x \\
=\int_{S} \phi_{r}(t=T)\left(c_{r}-\tilde{c}_{r}\right)(t=T) d x-\int_{T-\Delta t}^{T} \int_{S}\left(-\partial_{t} \phi_{r}+R \phi_{r}\right)\left(c_{r}-\tilde{c}_{r}\right) d x d t \\
=\int_{S}\left\{\phi_{r}\left(c_{r}-\tilde{c}_{r}\right)\right\}(t=T-\Delta t) d x-\int_{T-\Delta t}^{T} \int_{S}\left(\partial_{t}\left(c_{r}-\tilde{c}_{r}\right)+R\left(c_{r}-\tilde{c}_{r}\right)\right) \phi_{r} d x d t \\
=\int_{S}\left\{\phi_{r}\left(c_{r}-\tilde{c}_{r}\right)\right\}(t=T-\Delta t) d x+e_{r}^{N_{T}},
\end{gathered}
$$

where $e_{r}^{N_{T}}=-\int_{T-\Delta t}^{T} \int_{S}\left(-\partial_{t} \tilde{c}_{r}-R \tilde{c}_{r}\right) \phi_{r} d x d t$. Note we have used the equation for $c_{r}$ to eliminate some terms. If the diffusion and reaction grids are different, then some interpolation error is introduced that may be accounted for here. Note that

$$
\begin{gathered}
\left\{\phi_{r}\left(c_{r}-\tilde{c}_{r}\right)\right\}(t=T-\Delta t)=\phi_{r}(t=T-\Delta t)\left(c_{r}(t=T-\Delta t)-\tilde{c}_{d}(t=T)\right) \\
+\phi_{r}(t=T-\Delta t)\left(\tilde{c}_{d}(t=T)-\tilde{c}_{r}(t=T-\Delta t)\right) \\
=\left\{\phi_{d}\left(c_{d}-\tilde{c}_{d}\right)\right\}(t=T)+\phi_{r}(t=T-\Delta t)\left(\tilde{c}_{d}(t=T)-\tilde{c}_{r}(t=T-\Delta t)\right) .
\end{gathered}
$$

We insert this result above and perform the analogous operations for the diffusion substep. The result is, after integration by parts,

$$
\begin{aligned}
e_{d i s c} & =e_{r}^{N_{T}}+e_{d}^{N_{T}}+\int_{S} \phi_{r}(t=T-\Delta t)\left(\tilde{c}_{d}(t=T)-\tilde{c}_{r}(t=T-\Delta t)\right) d x \\
& +\int_{S}\left\{\phi_{d}\left(c_{d}-\tilde{c}_{d}\right)\right\}(t=T-\Delta t) d x, \\
e_{d}^{N_{T}} & =-\int_{T-\Delta t}^{T} \int_{S}\left(-\partial_{t} \tilde{c}_{d}+D \Delta \tilde{c}_{d}\right) \phi_{d} d x d t \\
& +\sum_{\mathcal{K} \in \Omega_{h}} \int_{T-\Delta t}^{T} \int_{\partial \mathcal{K}^{+} \backslash \partial \Omega}\left(D \phi_{d}\left(\hat{n}_{\mathcal{K}} \cdot \nabla\left[\tilde{c}_{d}\right]\right)-D\left(\hat{n}_{\mathcal{K}} \cdot \nabla \phi_{d}\right)\left[\tilde{c}_{d}\right]\right) d(\partial S) d t \\
& -\int_{T-\Delta t}^{T} \int_{\partial S} \hat{n} \cdot \nabla \phi_{d}\left(g_{d}-\tilde{c}_{d}\right) d(\partial S) d t .
\end{aligned}
$$

The advection grid may also be different from the diffusion grid, hence we insert

$$
\begin{gathered}
\left\{\phi_{d}\left(c_{d}-\tilde{c}_{d}\right)\right\}(t=T-\Delta t)=\phi_{d}(t=T-\Delta t)\left(c_{d}(t=T-\Delta t)-\tilde{c}_{a}(t=T)\right) \\
+\phi_{d}(t=T-\Delta t)\left(\tilde{c}_{a}(t=T)-\tilde{c}_{d}(t=T-\Delta t)\right) \\
=\left\{\phi_{a}\left(c_{a}-\tilde{c}_{a}\right)\right\}(t=T)+\phi_{d}(t=T-\Delta t)\left(\tilde{c}_{a}(t=T)-\tilde{c}_{d}(t=T-\Delta t)\right)
\end{gathered}
$$


and perform the analogous steps for the advection data, resulting in:

$$
\begin{aligned}
e_{d i s c} & =e_{r}^{N_{T}}+e_{d}^{N_{T}}+e_{a}^{N_{T}}+\int_{S} \phi_{r}(t=T-\Delta t)\left(\tilde{c}_{d}(t=T)-\tilde{c}_{r}(t=T-\Delta t)\right) d x \\
& +\int_{S} \phi_{d}(t=T-\Delta t)\left(\tilde{c}_{a}(t=T)-\tilde{c}_{d}(t=T-\Delta t)\right) d x \\
& +\int_{S}\left\{\phi_{a}\left(c_{a}-\tilde{c}_{a}\right)\right\}(t=T-\Delta t) d x, \\
e_{a}^{N_{T}} & =-\int_{T-\Delta t}^{T} \int_{S}\left(-\partial_{t} \tilde{c}_{a}-\nabla \cdot\left(\mathbf{v} \tilde{c}_{a}\right)\right) \phi_{a} d x d t \\
& +\sum_{\mathcal{K} \in \Omega_{h}} \int_{T-\Delta t}^{T} \int_{\partial \mathcal{K}+\backslash \partial \Omega}\left(-\left(\mathbf{v} \cdot \hat{n}_{\mathcal{K}}\right) \phi_{a}\left[\tilde{c}_{a}\right]\right) d(\partial S) d t .
\end{aligned}
$$

Interpolation between advection and reaction grids is taken into account:

$$
\begin{aligned}
&\left\{\phi_{a}\left(c_{a}-\tilde{c}_{a}\right)\right\}(t=T-\Delta t)=\left\{\phi_{a}\left(c_{a}-\tilde{c}_{r}\right)\right\}(t=T-\Delta t) \\
&+\left\{\phi_{a}\left(\tilde{c}_{r}-\tilde{c}_{a}\right)\right\}(t=T-\Delta t) \\
&=\left\{\phi_{r}\left(c_{r}-\tilde{c}_{r}\right)\right\}(t=T-\Delta t)+\left\{\phi_{a}\left(\tilde{c}_{r}-\tilde{c}_{a}\right)\right\}(t=T-\Delta t) .
\end{aligned}
$$

Insert this result in (3.14) to obtain

$$
\begin{aligned}
e_{\text {disc }} & =e_{r}^{N_{T}}+e_{d}^{N_{T}}+e_{a}^{N_{T}}+e_{\text {interp }}^{N_{T}}+\int_{S}\left\{\phi_{r}\left(c_{r}-\tilde{c}_{r}\right)\right\}(t=T-\Delta t) d x, \\
e_{\text {interp }}^{N_{T}} & =\int_{S} \phi_{r}(t=T-\Delta t)\left(\tilde{c}_{d}(t=T)-\tilde{c}_{r}(t=T-\Delta t)\right) d x \\
& +\int_{S} \phi_{d}(t=T-\Delta t)\left(\tilde{c}_{a}(t=T)-\tilde{c}_{d}(t=T-\Delta t)\right) d x \\
& +\int_{S}\left\{\phi_{a}\left(\tilde{c}_{r}-\tilde{c}_{a}\right)\right\}(t=T-\Delta t) d x .
\end{aligned}
$$

Finally, we repeat the above steps over the other time intervals, moving sequentially backward in time, and achieve the final equation

$$
\begin{aligned}
e_{d i s c} & =e_{A}+e_{D}+e_{R}+e_{\text {interp }}+e_{I C}, \\
e_{A} & =\sum_{i=1}^{N_{T}} e_{a}^{i}, \quad e_{D}=\sum_{i=1}^{N_{T}} e_{d}^{i}, \quad e_{R}=\sum_{i=1}^{N_{T}} e_{r}^{i}, \\
e_{\text {interp }} & =\sum_{i=1}^{N_{T}} e_{\text {interp }}^{i}, \quad e_{I C}=\int_{S}\left\{\phi_{s}\left(c_{0}-\tilde{c}\right)\right\}(t=0) d x .
\end{aligned}
$$

In practice it is desirable to write separate code modules to calculate the advection, diffusion and reaction error contributions. There are two reasons. One is because each of these error calculations may be independently verified 
as discretization error calculations for their respective sub-problems. This includes the adjoint solver and the quadrature for the local error calculations. The second reason is that having localized control over the adjoint solver may help to optimize the error calculation itself, since the resolution of the adjoint solution affects the quality of the error estimates. We note that interpolation amounts to a simple change of deterministic basis. Thus the initial condition and interpolation error contributions do not require a special treatment for UQ and are relatively easy to verify.

The advection, diffusion and reaction adjoint modules and error calculations use only the uncertain parameters for their respective sub-problems. Therefore the flexible-UQ framework is applied to calculate the global PC expansions for the primal and adjoint solution data and to use this data within the error calculation modules.

\section{Computational Example}

The numerical error may exhibit a complex distribution of values with respect to the uncertain problem data. As a result, the effects of numerical error with uncertainty in problem data upon the QoI distribution may be both surprising and significant. The following computational examples serve to illustrate the way that error and uncertainty may affect each other.

\subsection{Advection-diffusion without reaction}

In this example there will be no reaction component; equivalently set $R=0$ with no uncertainty in that value. We impose homogeneous Dirichlet boundary conditions for the advection-diffusion problem. These boundary conditions are also chosen for the split diffusion problem and for the advection problem at the inflow boundary. The spatial domain is $S=[0,1]^{2}$ and the time interval is $t \in[0,1 / 2]$. The domain $S$ is partitioned into uniform grids of square cells with respect to which we apply finite volume methods.

The advection direction is independent of space. The advection operator is discretized using a second-order upwind method with piece-wise linear, unlimited sub-grid slope reconstruction to defined values at cell faces. Time stepping is implemented using the explicit 4-stage Runge-Kutta method. This method is applied to both the primal and adjoint advection problems.

The diffusion modules are derived by using a centered second-order stencil for the Laplacian discretization with Crank-Nicholson time stepping. Thus the overall discretization error for the operator-split methods are second-order in space and time. The splitting method is as described previously, which is nominally first-order with respect to the associated time step.

The QoI is defined by the generalized Green's function

$$
\psi(x, y)=\frac{64}{49}(1+\cos (10 \pi(x-0.7)))^{4}(1+\cos (10 \pi(y-0.8)))^{4}
$$


for $(x, y):|x-0.7|<0.1$ and $|y-0.8|<0.1$ and zero otherwise. This acts as a local, weighted average of the solution centered at the point $(x, y)=(0.7,0.8)$ and results in a fairly smooth adjoint solution with the desired adjoint boundary conditions (zero Dirichlet). The point is to illustrate the importance that the distribution of numerical errors may have on understanding model outputs. We choose a grid with 40 cells in each direction and 18 time steps. The advection and diffusion grids are the same so that $e_{\text {interp }}=0$.

First we set $D=0.001$ and $\mathbf{v}=<0.5,1.0>$ with no uncertainty in the values and look at the error budget. The results, which are purely deterministic, are shown in Table 1. We include the usual approximation to the QoI, but we also include the result of using the numerical error to correct this value, denoted by $\mathcal{M}(c)$. Note that the errors are at least two orders of magnitude smaller than the size of the QoI value.

\begin{tabular}{|c|c|}
\hline $\mathcal{M}(\tilde{c})$ & $2.4293 E-1$ \\
$e_{\mathcal{M}}$ & $-1.6468 E-3$ \\
$e_{\text {split }}$ & $5.6912 E-5$ \\
$e_{A}$ & $-1.7311 E-3$ \\
$e_{D}$ & $2.5004 E-5$ \\
$e_{I C}$ & $2.3796 E-6$ \\
$\mathcal{M}(c)$ & $2.4128 E-1$ \\
\hline
\end{tabular}

Table 1: Error budget without parameter uncertainty.

In Table 2 the test is repeated except the $y$-component of the advection direction is taken to be in $\mathcal{U}(0.8,1.2)$. The expected values and standard deviations are shown for each quantity. The results differ dramatically from those of Table 1. The mean of the approximate QoI is significantly different from the deterministic case and it is unsettling that the variation in the QoI is so large. Upon examining the error, we see that the numerical error is the culprit, and moreover it is error from the advection module that is mostly to blame. After using the error values to correct the output a reasonable model is obtained; the mean is much closer to that of the deterministic case and the variation is much less. Thus accounting for the numerical error has made a significant difference in this problem.

\begin{tabular}{|c|c|c|}
\hline & $E[\cdot]$ & $\sigma[\cdot]$ \\
\hline $\mathcal{M}(\tilde{c})$ & $1.5418 E-1$ & $2.1229 E-1$ \\
$e_{\mathcal{M}}$ & $1.0300 E-1$ & $2.3567 E-1$ \\
$e_{\text {split }}$ & $1.7788 E-2$ & $3.9601 E-2$ \\
$e_{A}$ & $7.9254 E-2$ & $1.7391 E-1$ \\
$e_{D}$ & $9.9147 E-3$ & $2.2170 E-2$ \\
$e_{I C}$ & $2.3407 E-6$ & $3.1254 E-7$ \\
$\mathcal{M}(c)$ & $2.5718 E-1$ & $3.4981 E-2$ \\
\hline
\end{tabular}

Table 2: Error budget with parameter uncertainty. 


\subsection{Advection-diffusion-reaction}

The differences between this example and the previous example in 4.1 are that: we use 12 time steps and 10 grid cells in each direction; the PCE is of order 5 ; and we set the parameters for reaction, diffusion and the $y$-component of advection to be in $\mathcal{U}(0.8,1.0)$.

\section{Acknowledgements}

This research was funded by U. S. Department of Energy Office of Advanced Scientific Computing Research Applied Mathematics Program and performed under the auspices of the U.S. Department of Energy by Lawrence Livermore National Laboratory under Contract No. DE-AC52-07NA27344.

\section{References}

[1] Xiao Chen, Brenda Ng, Yunwei Sun, and Charles Tong, A flexible uncertainty quantification method for linearly coupled multi-physics decomposition: applications in reactive transport solved by operator-splitting, submitted, J. Comput. Phys., (2013).

[2] J. M. Connors, J. W. Banks, J. A. Hittinger, And C. S. WoodWARD, Quantification of errors for operator-split advection-diffusion calculations, tech. report, Lawrence Livermore National Laboratory, LLNL-JRNL566435, submitted, 2012.

[3] Jeffrey M. Connors, Jeffrey W. Banks, Jeffrey A. Hittinger, AND CAROL S. WoODWARD, A method to calculate numerical errors using adjoint error estimation for linear advection, submitted, SIAM J. Numer. Anal., 51 (2013), pp. 894-926. 